\title{
Research on Design and Application of Self-made Simulation Test Platform for University Lab
}

\author{
P. Zhang, Y. Zhang, and Y. Chang
}

\begin{abstract}
This paper will discuss how to design and assemble a simulation test platform, which is based on the university laboratory project, aimed at promoting both the quality of teaching and scientific research level in the university laboratory, and it can meet ergonomics requirements in terms of sitting position and vehicle driving comfort .In order to help students understand the property and distribution of the parameters of ergonomics, it is necessary to develop their abilities to analyze and solve problems. It can also stimulate their interests and enhance their creativity.
\end{abstract}

Index Terms-Ergonomics, self-made devices, simulation test platform, university laboratory.

\section{INTRODUCTION}

Experimental teaching is an important part of the university practice teaching system, which is an important part of cultivating students' application ability .Self-made experimental platforms can simulate the different states of operating and research on the integration of the man-machine interface, to verify and optimize ergonomics design.

Those have been referred in some relevant theoretical researches. One of researches was conducted by H. Yang, G.H. Li. They discussed the function of self-made experimental equipment of the university teachers in experimental teaching reform and 1aboratory construction in the "Promoting experimental teaching reform by Self-made experimental equipment". According to the experimental platform of biomechanics behavior of drivers, E. Zhang, J. Hong, W.W. Wu, and J. Liang thought they can simulate and analyze people's behavior in different driving environments and discuss how to match to the interface in the paper of "Design and analysis of a test stand for biomechanics of man-machine interface in automobile driving". The paper "The influence of chair design on teenagers scoliosis" referred to the difference body size between teenagers and adults, as well as the influence of action fields on daily behavior and daily sitting posture, F. Wang, X. Feng got the design of the chair suitable for teenagers in this age. And then, by virtual simulation technology, to determine whether the shape of seat is fitting human sitting posture or not, finally obtained the most effective data about the sitting posture.

Currently, all most of the experimental platforms are complex, multifunctional and expensive; but they do not

Manuscript received May 30, 2017; revised August 4, 2017. This work was supported in part by Hefei University of Technology under Grant SY201523.

The authors are with Hefei University of Technology, Hefei, China (e-mail: zhangp163@hfut.edu.cn, 1030331643@qq.com, 1343033530@qq.com). suitable to university laboratory. Therefore, this paper will introduce the design and manufacture of simulation test platforms. The benefits are low-cost, easy assembling, wide universality, which meet the needs of experimental teaching well. With the help of the ergonomic evaluation system, the ergonomics design can be verified and optimized.

\section{Design AND Assemble of Simulation Test Platform}

\section{A. Analyzing of Human Comfort in Sitting}

Sitting is one of the more relaxed positions in the body [1], which is beneficial to the circulation of the blood and reduces the feeling of fatigue in the body, and also helps the operator to take a stable posture for all kinds of delicate tasks. However, there are many adverse factors to human body health, such as abnormal bending of the spine, human pressure on the surface of the main support will produce discomfort [2]. Therefore, how to build a comfortable sitting position environment are an important aspect of ergonomics research and the basic content of project research.

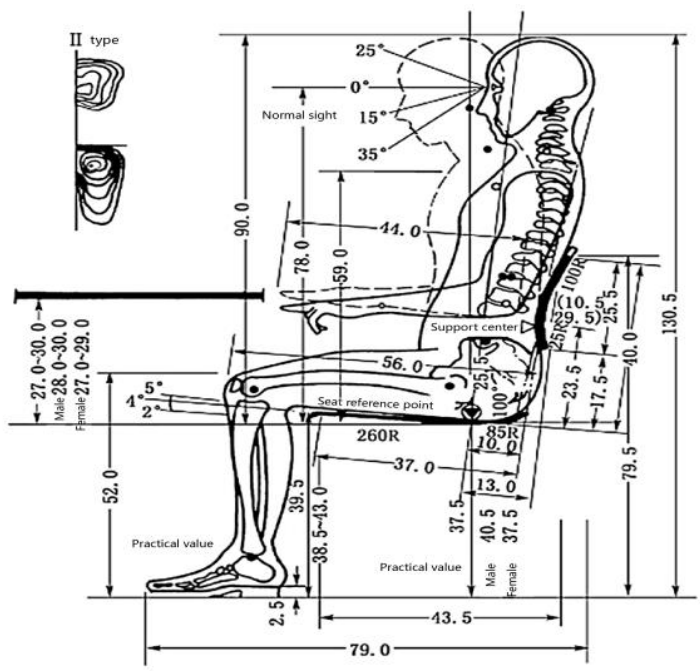

Fig. 1. Body sitting state.

\section{B. Design Principles of Simulation Test Platform}

The simulation test platform based on seated work space is composed of two parts: sitting test platform and automobile seat test platform. The seating test platform can be used to adjust the overall height, seating angle, sitting depth, back height, backrest angle and other parameters in order to meet the requirements of different sizes, different sitting postures and different working space. In addition, the platform can replace all kinds of seat moulds according to different needs. Hence, the seating test platform should have functional structure, supporting structure, installation point and 
adjusting mechanism. Among them, the backrest assembly and cushion assembly according to the 95-degree Asian men's thighs and spine-related ergonomic size to determine the size of the structure, including hard bearing structure and soft fill material. Work surface area should meet the 95-degree Asian male desk operating requirements [3]-[5]. All functional structures should be based on different test requirements in different sizes or materials to set a variety of programs, which are chosen to use in the test. The supporting structure is the overall frame of the test bed, including the ground-based load frame based on the basic form of the seat, the reference frame for the functional structure installation, and the institutional settings that meet the regulatory requirements. Functional structure should be installed in a proper manner to the supporting structure, so according to the functional structure of the force analysis to set a reasonable installation, the number of installation points and location. Different types of cushion assembly should have the same installation structure. Likewise, different types of cushions and worktops should also have the same mounting structure. Finally, the adjustment mechanism of the office seat test platform is set according to the following parameters: sitting height, sitting depth, backrest height, backrest angle, table height, table angle.

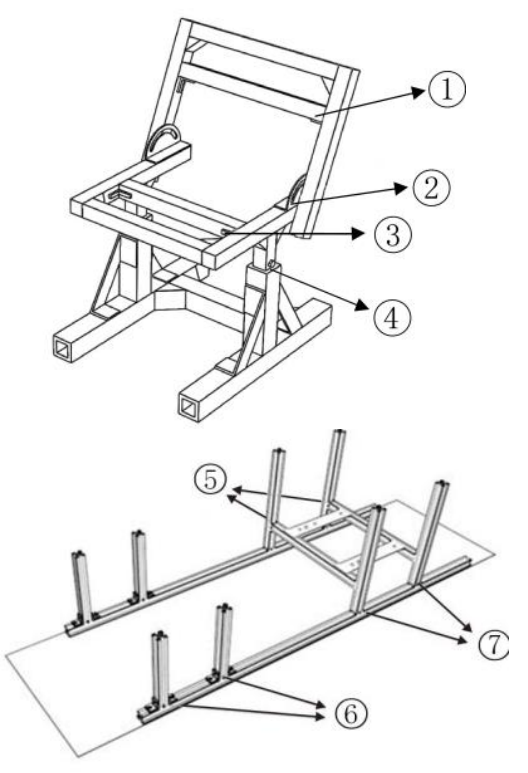

Fig. 2. Prototype of the experimental platform.

Vehicle driving simulation test platform should have some parts of function structures, support structures, installation and adjustment mechanism. First of all, the functional structure includes car seat assembly, steering wheel assembly and driving pedal assembly. This professional independent equipment, including seat, steering wheel and pedals, are custom made subject to manufacture requirements. Then, the supporting structure is the whole framework of the test platform, including the bearing frame of vehicle seat, the bracket of steering wheel and the position reference of car pedal. To be ensured the reliability of the test operation, the reasonable installation methods, positions and quantities can be set up by the stress analysis of the functional structure. The same installation forms are shared by different types of the same components, which is easy to replace during the test. Finally, the regulators should be set up on the basis of the following parameters: the height of the seat; the height, the angle and the distance of the steering wheel; the angle and the distance of the automobile pedal.

In order to meet those requirements mentioned above, the projects of the prototypes of the office seat test platform and the vehicle simulation test platform are demonstrated in Fig. 2 .

\section{The Assembly of Simulation Test Platform}

\section{1) Building supporting structures}

We choose aluminum sections which label is $4040 \mathrm{~L}$ as main elements to building the test platform. Aluminum profile has light mass, and is easy to operate, may ensure the whole structure stable, reliable, and easy to disassemble (Fig. 3). Some joint elements were chosen to fastening the structure, like the corner fittings, $\mathrm{T}$ bolts, and flange nuts. The authors of the accepted manuscripts will be given a copyright form and the form should accompany your final submission.
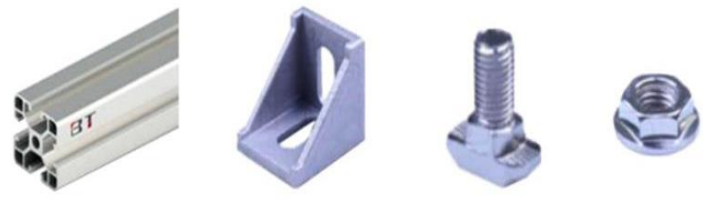

Fig. 3. Aluminum sections and joint elements.

\section{2) Setting up adjustment structures}

There are four kinds of adjustment mechanisms in the automobile seat test platform the mechanism in which the position of part (1), part (3) is used for the position adjustment, the position at the position of the base part (4) is used for height adjustment, and the mechanism at the position of part (2) is used for angle adjustment. There are three adjustment mechanisms for the car seat test platform. Among them, part (5) is used for height adjustment, part (6) and part (7) are used for position adjustment. The function of the position adjustment mechanism and the function of the height adjustment mechanism at the position of part (5) are realized by changing the relative position of the profile. The height adjustment function at position part (4) is achieved by means of the profile telescopic kit. The angle adjustment mechanisms at the position of the part (2) use the angle dial fitting to realize the function of angle adjustment.

\section{3) Setting up the way of installation}

In order to ensure the replacement of different types of functional structure on the test platform, the same functional structure using the same installation. In the car seat test platform, the cushion assembly and the backrest assembly are fixed by two bolts embedded in them and two bolts on the support structure. In addition, the car seats need to be assembled with two of the profiles, each of which is fixed by two bolts. The steering wheel and the pedal are fixed by a custom pipe, placed directly on the ground.

D. Design of Healthy Learning Chair Which Adjusting the Physiological Characteristics of Teenagers 


\section{Dynamically}

The design targets mainly focus on domestic teenagers. By the theory of ergonomics, the healthy learning chair, which embodies physical characteristics, and psychological characteristics of teenagers, is suitable for teenagers to keep learning posture well. This chair is intelligent in some degree, to achieve automatic adjustment to match the posture of the user, and to help parents give the healthy guidance to teenagers, to help them improve learning efficiency [6].

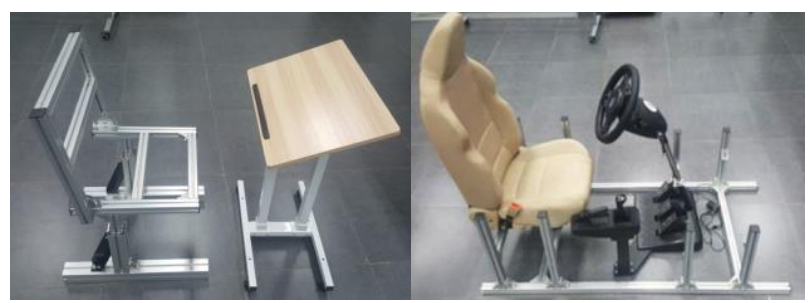

Fig. 4. Test platform.

\section{1) Adjustment design of chair}

As we know, students' body sizes and learning postures are different from different ages, according to the physiological characteristics of them; we plan to choose adjustable dimensions for the chair, such as the height, the depth and the backrest, et al. (Fig. 5).

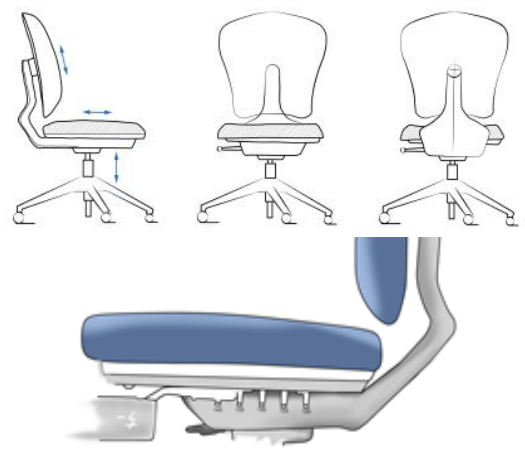

Fig. 5. Adjustable dimensions of the chair: height, depth and backrest height.

The range of female's height can be included in the data of male, the data changes from $1.381 \mathrm{~m}$ to $1.839 \mathrm{~m}$. According to the ergonomics standard, chair height should be between $300 \mathrm{~mm}-480 \mathrm{~mm}$, adjustment range in $180 \mathrm{~mm}$.

At the same time, as the height increases, the chair depth should be increased accordingly, similarly the chair depth can be adjusted in $150 \mathrm{~mm}$. The height of the lumbar pad affects the comfort of the user directly, depending on the deferent heights, the lumbar pad height adjustment range in $80 \mathrm{~mm}$ [7].

\section{2) Back design of chair}

By the structure of human spine, spine bending range is limited greatly. In fact, the adjacent two pieces of vertebrae can only change a small angle and must change smoothly. They can be approximated as discrete points of a continuous function. However, there is not a public "spine curve" which is suitable to all, because the curve of the spine is different from the posture changing of the people, while the dimensions are not the same by people. Through researching a large number of documents, finally the Bezier curve is selected to match the design requirements.

Fig. 6 shows the chairs back which simulate the human body curve by using the Bezier curve to fit and transit smoothly. The back of chair fits body structure of oriental teenagers, it can provide convenient lumbar support, make the spine to maintain coordination, avoid back pain, and reduce fatigue when students are working, reading, writing, or resting.

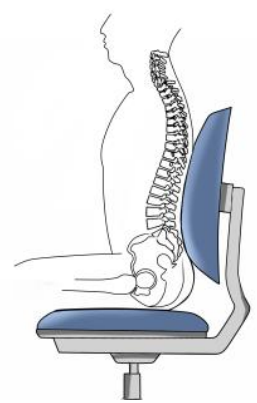

Fig. 6. Bezier curve fitting chair back.

\section{3) Chair design of multi module cushion and backrest}

This chair cushion can provide multi point movable supporting. The main parts of chair cushion and backrest are made of modular structure. The cushion is divided into 6 modules, and 3 modules in the lumbar pad. The connection between the modules is filled with springs and some materials which neither too hard, nor too soft. Each module is equipped with automatic driving device that can adjust the angle and position of the module at any time to realize the transformation of different forms of cushion, in order to fit the users' back and buttocks perfectly. By the control system, the position and the shape of the cushions are automatically adjusted, and similarly to the lumbar pads. So as to realize the function that the chair can adjust the posture of the user actively, as shown in Fig. 7-Fig. 8.

In the process of application, the chair cushions and the lumbar pads are set two modes:

\section{a) Manual mode}

After selecting this mode, the user can adjust the height and position of each module through the control buttons, until making themselves comfortable.

\section{b) Auto-cycle mode}

The system presets three forms of chair cushion. After selecting this mode, the control system will adjust cushion shape to the next state automatically every certain period of time, to make people change a posture unconsciously. By changing the center of gravity of the human body, it can reduce fatigue when teenagers are working for a long time [7].

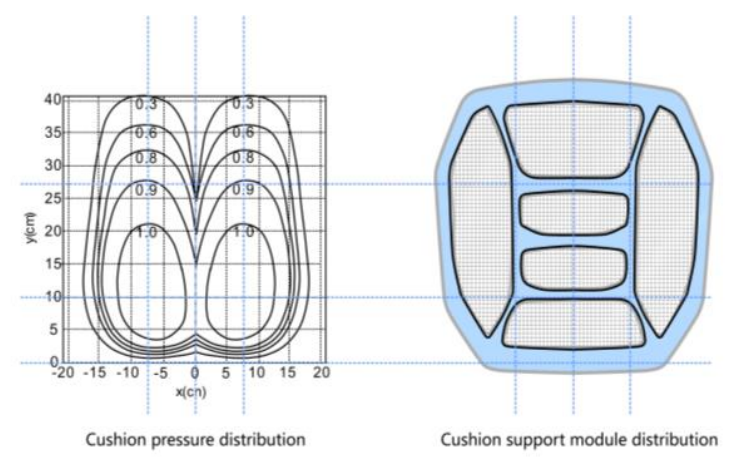

Fig. 7. Diagram of multi module cushion. 


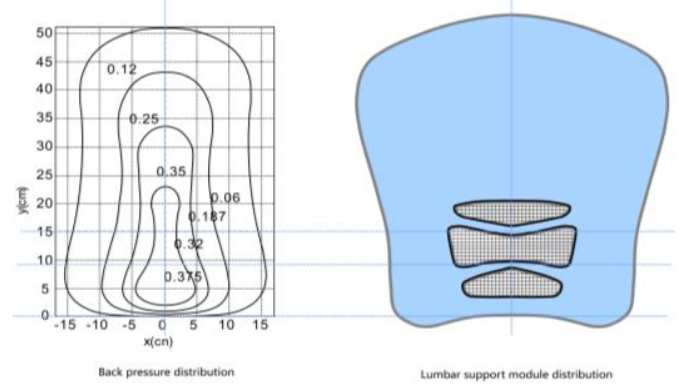

Fig. 8. Diagram of multi module lumbar pad.

\section{Application of the Simulation TeSt PlatForm}

Ergonomic simulation test platform of sitting work space can simulate many different working stations. In the course of the experiment, students use the human body static size, sitting physiology, mechanics and other ergonomic related knowledge, combined with body pressure distribution test and sEMG tester to adjust the seat height, depth, backrest angle and relative position and other operating equipment of the scale parameters. Students will be able to calibrate the test object and seek the optimal program parameters, from the operation convenience, comfort, security of these three aspects.

\section{A. Application Environment of Human-Machine} Function Simulation Experiment Platform

\section{1) Supervise and control the working environment}

At present, the monitoring center has been basically intelligent and electronic, control personnel receive signals and signal processing through the computer terminal. At same time computer terminal intuitive signal to the control personnel through the display and voice equipment. The control personnel transmit and feed the signal by controlling the original. General control center of the control equipment mainly include: workstations, work seats, display, telephone fax machines, call equipment, control components, related text materials and spare parts storage box.

\section{2) Driving station of the car}

In the design of the car seat, the man-machine form design focuses on the action and physical needs of the occupants. Among them, the physiological needs, including human comfort and health and other factors. The car driving simulation test bed can simulate the car driving environment .In the course of the experiment, students use the human body static size, sitting physiology, mechanics and other ergonomic related knowledge, combined with body pressure distribution test and sEMG tester to adjust the seat height, depth, backrest angle and relative position and other operating equipment Of the scale parameters. Students will be able to calibrate the test object and seek the optimal program parameters, from the operation convenience, comfort, security of these three aspects. The platform can simulate the monitoring environment, and then study the control personnel's sitting work environment when they are working.

\section{B. Experimental Test of Man - machine Function Simulation Test.}

\section{1) Testing of the driver's hands touch interface and} operating comfort

\section{a) Experimental objectives}

Both the handles and operation buttons are basically required to reduce the frequency of incorrect operation to achieve safe driving [7]. The purpose of this experiment is to learn the structural functions, geometric parameters and seat design related ergonomic requirements of the office seats and car seats, and assess the comfort of the seat.

\section{b) Experimental contents}

The tester simulates the operating state of the operator during different operations (Fig.9-13).

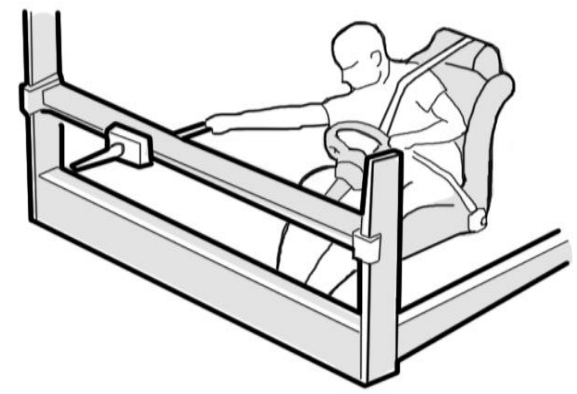

Fig. 9. Experimental process.

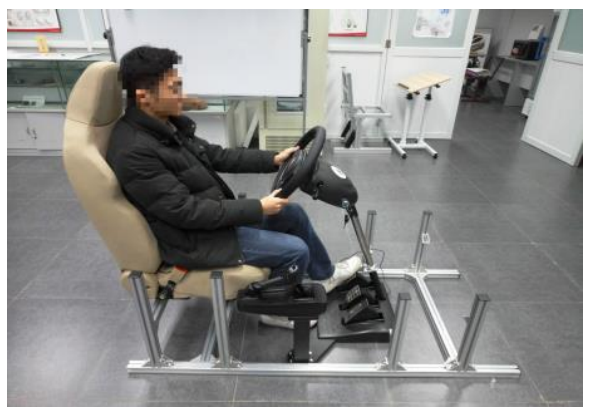

Fig. 10. Experimental process.

c) Analyzing the experimental results

Collection the statistics of how change the comfort level in the process of using. Then, through the measurement, reasonability of the placement should be checked based on data calculation and comparison. Compared with the experimental data, standard parameters of automobile driving comfort can be verified and the reasonability of the components will be examined as well.

\section{2) Testing of comfort of seats}

\section{a) Experimental objectives}

In the course of sitting, the comfort, safety and scientific design of seat should be taken into considerations. The purpose of this experiment is to help students understand the structures and functions of seat, study geometric parameters and ergonomic requirements in the design of the car seat, and evaluate the comfort of the seat.

b) Experimental contents

The subjects will simulate using ng state during the using process, and take different types of seats within a set period of time (Fig.13-14). After that, the body pressure data were recorded, and then factors that affect the comfort of seats can be analyzed. 


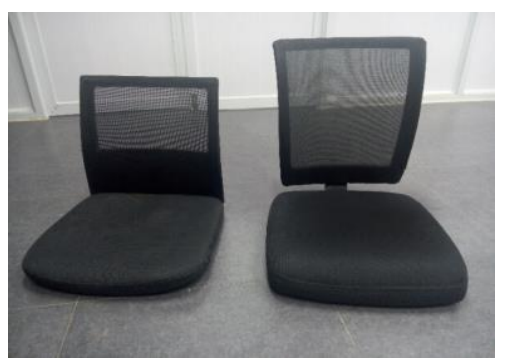

Fig. 11. Two seats by experiment.

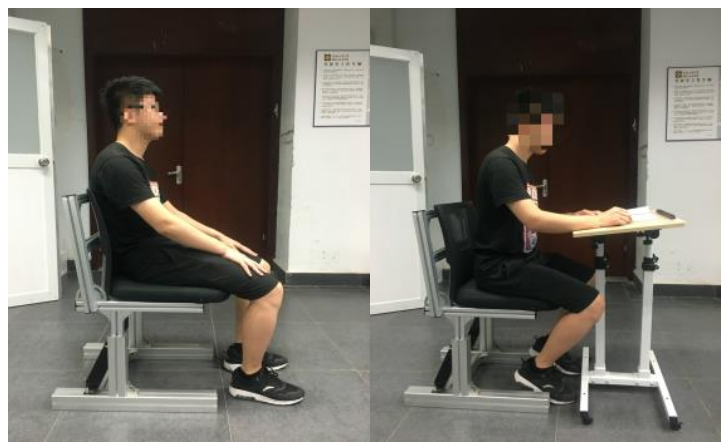

Fig. 12. Experimental process.

\section{c) Analyzing of experimental results}

By recording the area of contact, average pressure, and peak pressure and so on, then to calculate the maximum longitudinal pressure and pressure gradient by relevant professional software, and to draw the curve of pressure and isobaric map. Through analyzing these experimental data, the body pressure distribution of three car seats can be obtained, and the design of seat will be optimized, which provides a theoretical basis of the comfort evaluation of seat [8], [9].

\section{d) Testing of the Comfort of Sitting Posture}

\section{1) Experimental objectives}

It is the key factor that whether tester's motion is comfortable in the design of human-machine interface. The aim of this experiment is to study relevant knowledge of seat comfort requirements for drivers and to understand the influencing factors about human body size for product design, so as to guide the practical design.

\section{2) Experimental contents}

On the basis of the simulation test-platform, the simulated space can be built up. In the stimulation status, when the sitting position of tester was close to the threshold of fatigue, the data of sitting position, the pressure distribution of sitting and the change of the sitting posture were recorded and monitored (Fig. 13).

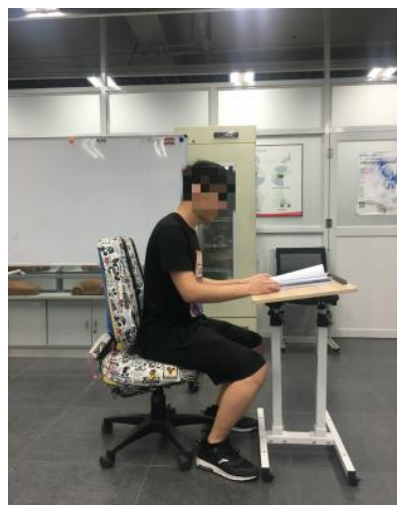

Fig. 13. The experimental scene.

\section{3) Analyzing of experimental results}

Analyzing statistically data of the sitting position and the pressure distribution; Recording the sitting posture change; Recording the psychological status change of the subjects; generally, analyzing these streams of data can get the threshold of fatigue and the distribution of pressure distribution at specific time. So we got the driving posture comfort data based on this experiment.

\section{CONCLUSION}

In this paper, designing and constructing a simulation test platform for university laboratory. In a sense, the test platform is simple and crude, but it has a lot of advantages in teaching practice. The simulation test platform can meet the requirements of using, so it can be used to verify the theory of the ergonomics. In the course of the experiment, it not only cultivate the students' practical skills and practical ability, but also has significance on the further enhance of ergonomics laboratory teaching and scientific research.

\section{ACKNOWLEDGMENT}

P. Zhang thanks to the funding and research conditions provided by Hefei University of Technology. The successful completion of this paper depends on researching achievement of the teaching research project of Hefei University of Technology, which is based on the project about ergonomics simulation test platform of sitting work.

\section{REFERENCES}

[1] Y. Wen, "The research of comfort of automobile seat based on ergonomics," M.S. thesis, Dept. Industrial Design, Hefei Univ. of Technology, Hefei, China, 2015.

[2] P. Z. Sun, "Research on the key comfortable indicators of office chair," M.S. thesis, Dept. Industrial Design, Hefei Univ. of Technology, Hefei, China, 2016.

[3] H. B. Yang, H. M .Jiang, and X. Wang. "Research of health office chair design based on ergonomics," Design, vol. 2, pp. 24-25, 2012

[4] Y. L. Ding, Ergonomics, Beijing: Beijing Institute of Technology Press, 2000, pp. 20-25.

[5] A. R. Tilley, The Measure of Man \& Woman: Human Factors in Design, Tianjin: Tianjin University Press, 2008. pp. 11-17.

[6] D. Y Luo, "The ergonomic concept in student chair design," Journal of Southwest Agricultural University (Social Sciences Edition), vol. 1, pp. 185-186. 2012.

[7] H. Li et al., "Height and weight standardized growth charts for Chinese children and teenagers aged 0 to 18 years," Chinese Journal of Pediatric, vol. 1, pp. 487-492. 2009.

[8] Q. Yuan, W. C. Liu, and S. Zhang, "Design and construction of multi parameter adjustable automobile human computer interface experiment platform," China Mechanical Engineering. vol. 10, pp. 1257-126, 2008.

[9] J. H. Chen et al., "A research on the evaluation of driver's posture comfort," Automobile Engineering. vol. 10, pp. 548-552. 2013.

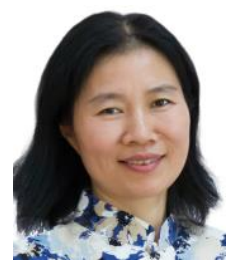

Ping Zhang was born in July 19, 1969 in Huaibei city, Anhui province China. She graduated from Hefei University of Technology in 1994, and received a master's degree in engineering, from school of mechanical engineering. She focused on the research field of product design and ergonomics design. In 2010-2011, she has been to University of California at Berkeley as a visiting scholar. During that time, she specialized in research on the theory and application of ergonomics in school of Bio mechanical engineering. At present, she is a doctoral student of Hefei University of Technology. Her research area is the design and manufacture of environmental consciousness. 
She has been engaged in teaching and research in industrial design for many years. She is currently an associate professor and master supervisor at the school of architecture and art at Hefei University of Technology. The main publications include: Industrial product modeling design (Beijing, China, China Machine Press, 2016); Ergonomics Applied Research on Virtual Ergonomics Applied Research on Virtual (Atlantis Press, 2015:145-148); Comfort Analysis of Automobile Seat Comfort of
Automobile Seats Based on 3D Human Models (Atlantis Press, 2015:393-395). She is interested in product design.

Prof. Ping is a senior member of Human Factors and Ergonomics Society (International Ergonomics Society); a director of industrial design branch of China Mechanical Engineering Society; A vice chairman of Anhui Industrial Design Association. 